\title{
Packet-selective photonic add/drop multiplexer and its application to ultrahigh-speed optical data networkings in LAN and MAN
}

\author{
Ken-ichi Kitayama ${ }^{1)}$ and Masayuki Murata ${ }^{2)}$ \\ Osaka University 85 \\ 1) Department of Electronics and Information Systems, 2-1 Yamadaoka, Suita, Osaka 565-0871, \\ Japan, (E-mail: kitayama@comm.eng.osaka-u.ac.jp) \\ 2) Cybermedia Center, 1-30 Machikaneyama, Toyonaka, Osaka 565-0043, Japan, (E-mail: \\ murata@ics.es.osaka-u.ac.jp)
}

Key words: packet transmission, asynchronous access, optical code correlation, performance modelling, optical implementation

\begin{abstract}
We propose packet-selective photonic add/drop multiplexing (P-ADM), which can determine the source and destination addresses in the packet header based upon optical code correlation and its novel application to ultrahigh-speed optical data networking in LAN and MAN. It is distinct from conventional wavelength ADM ( $\lambda$-ADM) having the wavelength granularity, in that P-ADM provides a packet granularity by individually handling optical packets on a single wavelength. As an application of P-ADM to optical data networking, a rate-controlled asynchronous access is studied, and the performance analysis and numerical examples are presented. The optical implementation of P-ADM is also presented, in which the photonic label selector, structured with a fiber Bragg grating (FBG), is a key to the ultrahigh-speed processing capability up to a few tens of Gpps (packet per second).
\end{abstract}

\section{INTRODUCTION}

There is a growing demand for high-bandwidth and differentiated data services. Given the inherently bursty nature of data traffic, the fixed-bandwidth TDM links is not an efficient solution. Optical data networking, which uses

The original version of this chapter was revised: The copyright line was incorrect. This has been corrected. The Erratum to this chapter is available at DOI: 10.1007/978-0-387-35491-0_28 
WDM links as the optical channels [1,2], is an alternative approach to the TDM-based data networking. Optical data networking eliminates unnecessary protocol stack in each network layer, thus drastically reducing the cost and complexity of networking $[3,4]$.

In optical data networking, the optical add/drop multiplexer (ADM), located at access nodes, will be key device. A wavelength add/drop multiplexer $(\lambda-A D M)$ enables each wavelength to either add/drop and electronically processes at the node or to optically bypasses the node electronics (Fig.1(a)). This capability of optically bypassing a node's electronic layer can be exploited to reduce overall electronic processing, resulting in the reductions of both the transfer delay and the number of electronic digital crossconnect switches and routers. $\lambda$-ADM can be adopted for different types of traffics such as static or dynamic circuit switching, packet switching, and flow switching [5]. However, $\lambda$-ADM is of granularity of wavelength, and this imposes a limit that it can only handle traffic on an optical channel, that is, a wavelength path, and it is unable to handle traffic on a packet-bypacket basis. This may waste the wavelength resource and limit the usage of optical channels. On the other hand, conventional SONET ADM has a finer granularity for handling traffic. It picks out low-speed streams from a highspeed stream and likewise, adds low-speed streams to a high-speed stream [6]. However, the speed of electronic processing will eventually become a bottleneck to the overall processing capability as the bit rate of the WDM link goes higher. Hence, there would be a growing demand for high-speed multi-functional ADM in the future optical data networking, which can handle traffic in finer granularity.

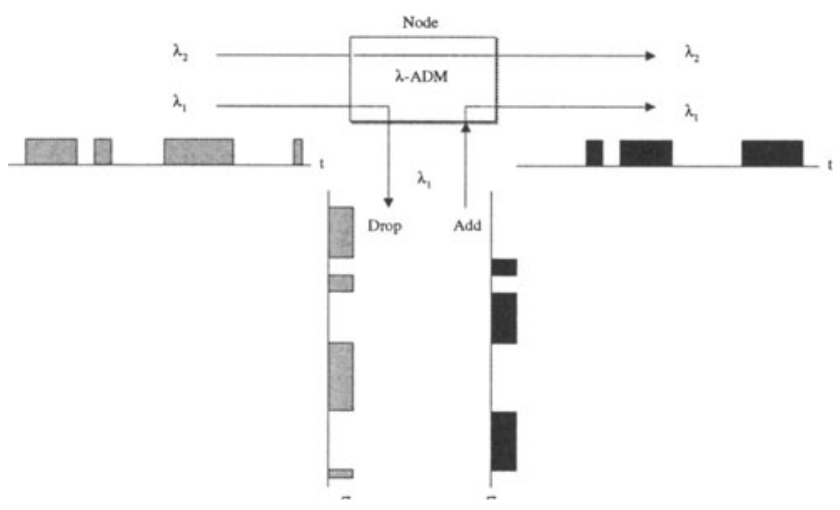

Fig. 1(a) Wavelength add/drop multiplexer ( $\lambda$-ADM) 
There have been a variety of optical packet switching schemes, in which the packet label are mapped in optical domain and processed optoelectronically [7-12]. Very recently, photonic router for Internet Protocol, IP packets has been proposed and demonstrated [13-15]. In the photonic IP router, the photonic labels are mapped onto a family of optical codes, which have been utilized optical code division multiplexing (OCDM) technique [16-18]. OCDM is a class of transmission and multiplexing techniques other than TDM, WDM, and space division multiplexing (SDM). OCDM inherits unique features from the wireless code division multiple access (CDMA) [19]. It was confirmed that as the encoding and decoding are performed in optical domain, the packet processing capability exceeds an order of gigapacket per second(Gpps).

In this paper, packet-selective photonic add/drop multiplexing (P-ADM), which can read the source and destination addresses in the packet header in optical domain, and its novel application to ultrahigh-speed optical data networking in LAN and MAN are proposed. It is distinct from conventional wavelength $\mathrm{ADM}(\lambda-\mathrm{ADM})$, in that P-ADM provides a packet granularity by individually handling optical packets on a single wavelength. In the application of P-ADM to optical data networking, a rate-controlled asynchronous access is studied, and the performance analysis and numerical examples are presented. The optical implementation of P-ADM is also presented, in which the photonic label selector, structured with a fiber Bragg grating (FBG), is a key to the ultrahigh-speed processing capability up to a few tens of Gpps (packet per second).

\section{CONCEPT OF PACKET-SELECTIVE PHOTONIC ADD/DROP MULTIPLEXER (P-ADM)}

\subsection{Principle of operation}

P-ADM is located at a node, and it drops to the node or bypasses the node the arriving packets or adds the generated packets from the node (Fig.1(b)). It handles traffic on a single WDM link on a packet-by-packet basis. The basic architecture is illustrated in Fig.2. The fundamental functions of PADM include photonic label selection and optical switching. The input and output ports are connected to a single WDM link. The photonic label in the header of arriving packet is processed, and according to the label the optical switch is driven by the control signal to direct the packet to the outgoing port either for dropping or bypassing. 


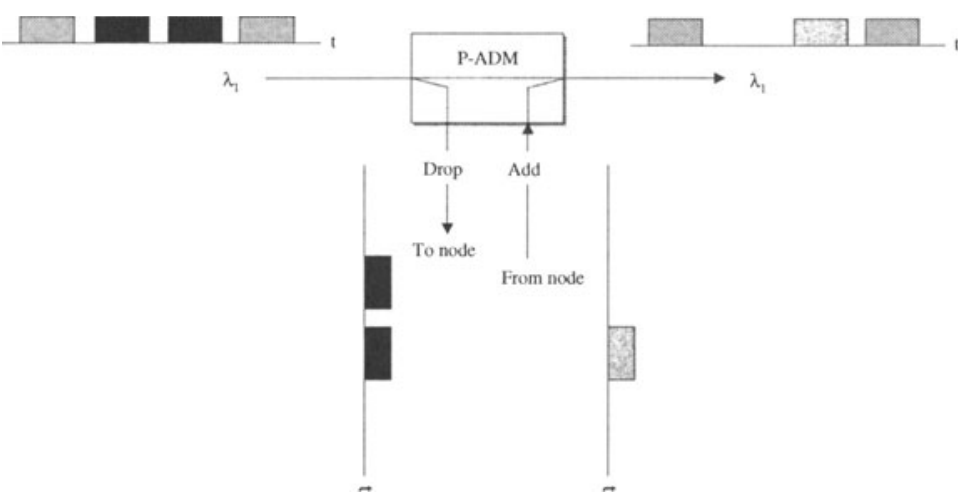

Fig. 1 (b) Proposed packet-selective photonic ADM (P-ADM)

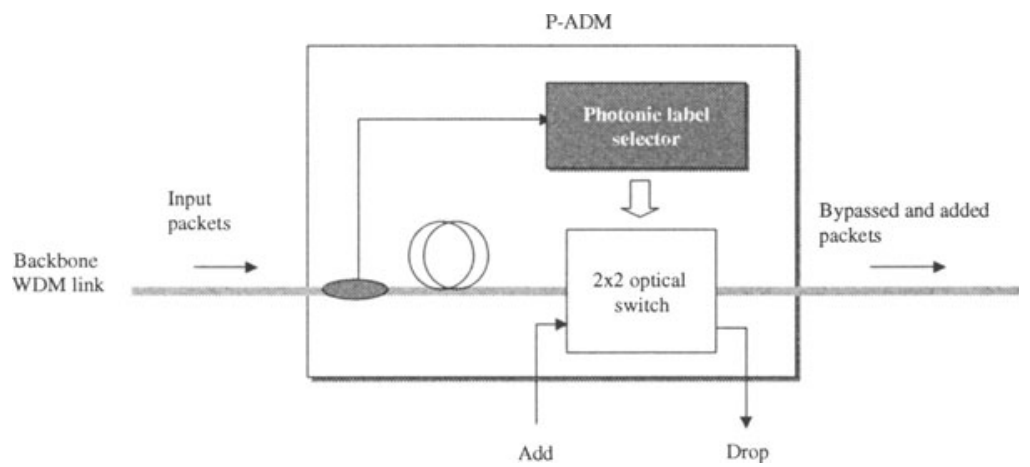

Fig.2 Basic architecture of packet-selective photonic ADM (P-ADM) module 


\subsection{Photonic label selector}

The optical packet is structured as shown in Fig.3. The photonic label in the header is simply a relatively short, fixed-length identifier that bears the information of the destination address and source address. At the end of the packet a flag that indicates the end must be attached. The other overhead information could be included in the label upon the request. The remaining network control information will be encapsulated in the frame of higher layers.

As shown in Fig.4 each node is uniquely assigned with a label, and each label is mapped onto an optical code [13]. The optical code is a sequence of optical pulses, so-called chip pulses. The chip itself is a short pulse, and the time duration of the sequence has to be accommodated within a bit time duration. The photonic label selection is based upon the optical correlation between the optical code of arriving packet and the assigned code of the node. The optical correlation is performed by matched filtering in time domain. For example, a family of optical codes of 4-chip long codes and their autoand cross-correlation waveforms are illustrated in Fig.4. The code is bipolar, in which the phase of optical carrier of individual chip pulse takes two states of either 0 or $\pi$, representing binary value of 1 or -1 , respectively. As increasing the number of nodes, the longer code will be required. Unique to the label selector is that no optical logic operation is involved, and this is a key to the ultrahigh-speed operation of P-ADM.

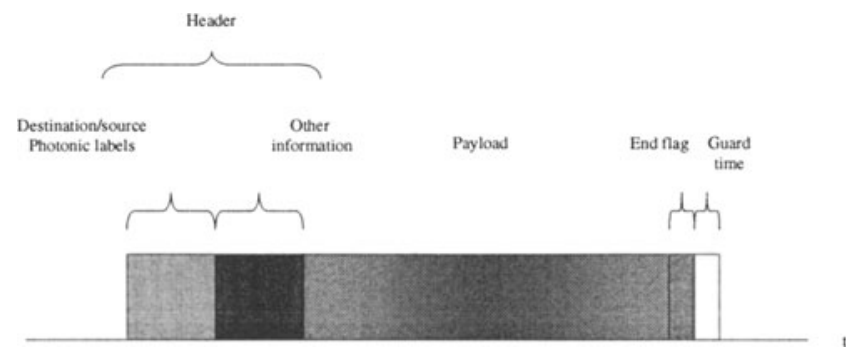

Fig.3 Optical packet 


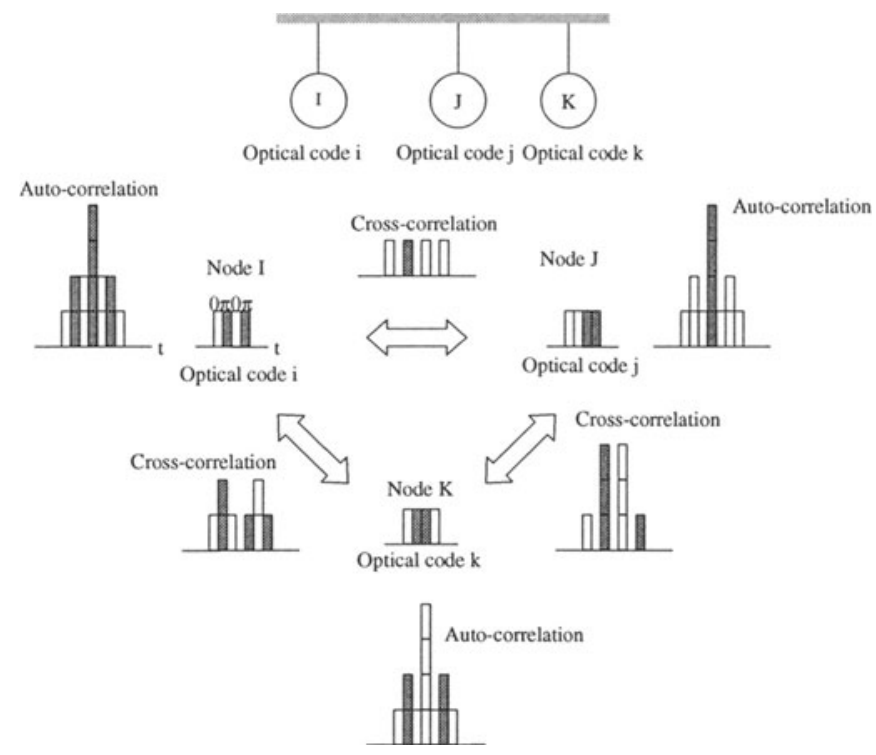

Fig.4 Address assignment to each node. A family of 4-chip long, bipolar optical codes and their auto- and cross-correlation functions

\subsection{Key building block: P-ADM module}

We will discuss the building blocks of P-ADM shown in Fig.2. First, the photonic label selector is structured with an optical correlator, a time-gate, an O/E conversion, and thresholding (Fig.5(a)). Promising device for the optical correlator is a fiber Bragg grating (FBG) (Fig.5(b)). An advantage is that a single FBG can select the photonic label as well as the wavelength, if the grating period is tuned to a desired wavelength. A super-structured FBG can generate bipolar optical codes [20]. The identical optical device must be used for the optical encoder.

Several high-speed off-the-shelf optical space switches have now become available. The switching speed of semiconductor optical amplifier (SOA) gate switch is in the sub-nanosecond regime, which is well beyond the requirement for the switching speed. Unfortunately, there is no random access optical memory, equivalent to electronic RAM. The optical fiber delay line is only an optical device that temporarily buffers an optical packet. 


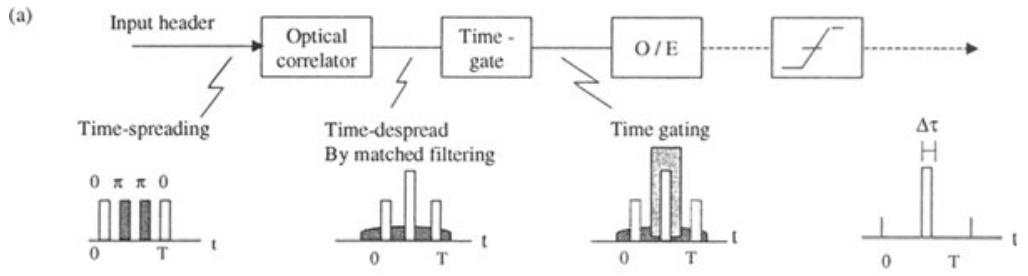

T: One bit time duration

(b)

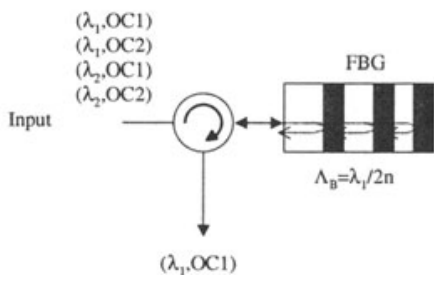

Fig.5 (a) Configuration of photonic code selector. (b) FBG-based optical correlator

\section{APPLICATION TO OPTICAL DATA NETWORK- INGS IN LAN AND MAN: LINE ACCESS BY RATE-CONTROLLED ASYNCHRONOUS PACKET TRANSMISSIONS}

\subsection{Operation mechanisms}

For data transmissions we want to allow the bursty transmission of packets in LAN and MAN, by which a flexible use of bandwidth is expected. For this purpose, we will propose a rate-controlled line access method, and we will implement this by using P-ADM. We use a leaky-bucket mechanism [21] to control the packet emission onto the line at each node.

The leaky-bucket is a virtual device to control the burstiness of the flow. As shown in Fig.6, tokens arrive at the token pool with rate $\rho$ and are buffered in the token pool if the token pool is not full. If an arriving packet finds the token pool nonempty, it departs the node instantaneously, and at the same time one token is removed from the token pool. The packet is discarded if an arriving packet finds the token pool empty. When a token is generated, and the packet buffer is nonempty, the packet on the top of queue departs the 
buffer immediately by removing one token. The depth of the token pool determines the burstiness of the flow; the larger size of the token pool allows more bursty packet transmissions.

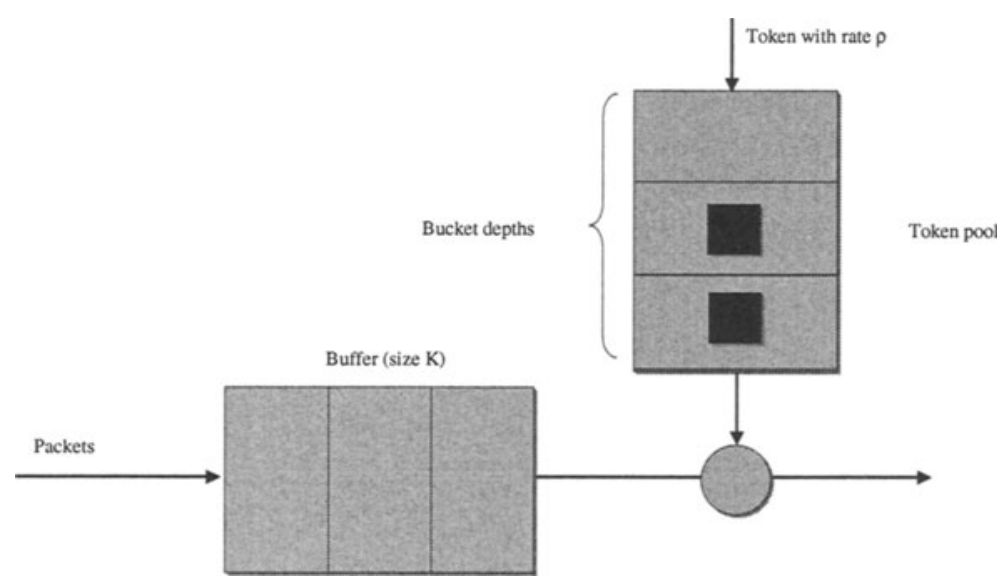

Fig.6 Logical structure of leaky bucket

\subsubsection{Rate control using leaky-bucket}

For a realistic scenario of the rate-controlled line access, we need to take account of the dynamics of the leaky-bucket. The leaky-bucket parameters should be determined for a given distribution of packet arrival rate. Since the packet buffer is finite, the packet loss probability should be estimated when determining the leaky-bucket parameters. For this purpose, we introduce $N$ and $C$ that represent the line capacity and the number of terminals connected to the line, respectively. Assuming that the packet length is fixed, the following procedure then determines the leaky-bucket parameters. Here, our simulation will forcus on the bus topology, however, P-ADM will also be applied to a ring topology.

1. Assume that the traffic load distribution is known. Let the mean rate be $\lambda_{i j}$ for the packet flow from node $i$ to node $j$, and let $\lambda_{i}=\sum_{j=i+1}^{N-1} \lambda_{i, j}$.

2. The admissible traffic load at each node is then determined by taking into account the relative use of the line capacity. In the case of the balanced traffic, it is simply determined as 


$$
C \times \frac{\lambda_{i}}{N^{2} / 4}
$$

where $N^{2} / 4$ is the number of connections on the most congested link. Note that other bandwidth allocation can also be considered by, e.g., considering the fair allocation of packet loss probabilities among nodes. For this purpose, however, we need to formulate some optimization problem.

3. The admissible load given by Eq.(1) determines the leaky-bucket parameter $\rho_{i}$ for node $i$.

4. Analyze the leaky-bucket to determine the packet loss probability and waiting time at the packet buffer for given packet arrival distributions.

Note that we need more strict arguments in performing above procedures. Those will be described in the next subsection by considering the case where the packet arrivals follow a Poisson distribution.

In the above, we still have a freedom in determining the burst size specification $\sigma_{i}$. For larger $\sigma_{i}$, the packet loss probability will decrease, which leads to the performance improvement. However, the upstream node $j$ is allowed the bursty packet then, bursty arrivals of packets from the upstream nodes inhibit the packet transmission at downstream node. Once we are given a permissible packet loss probability at each node, however, we can determine the bucket size and then obtain the maximum packet delay at the node in order.

\subsubsection{Analysis of leaky-bucket}

In this subsection, we present an analysis for the leaky-bucket at each node, which is a slight extension of the approach shown in [21]. Packets arrive at the packet buffer according to a Poisson process with rate $\lambda$. Note that we will omit the subscript representing the node number in the below. 


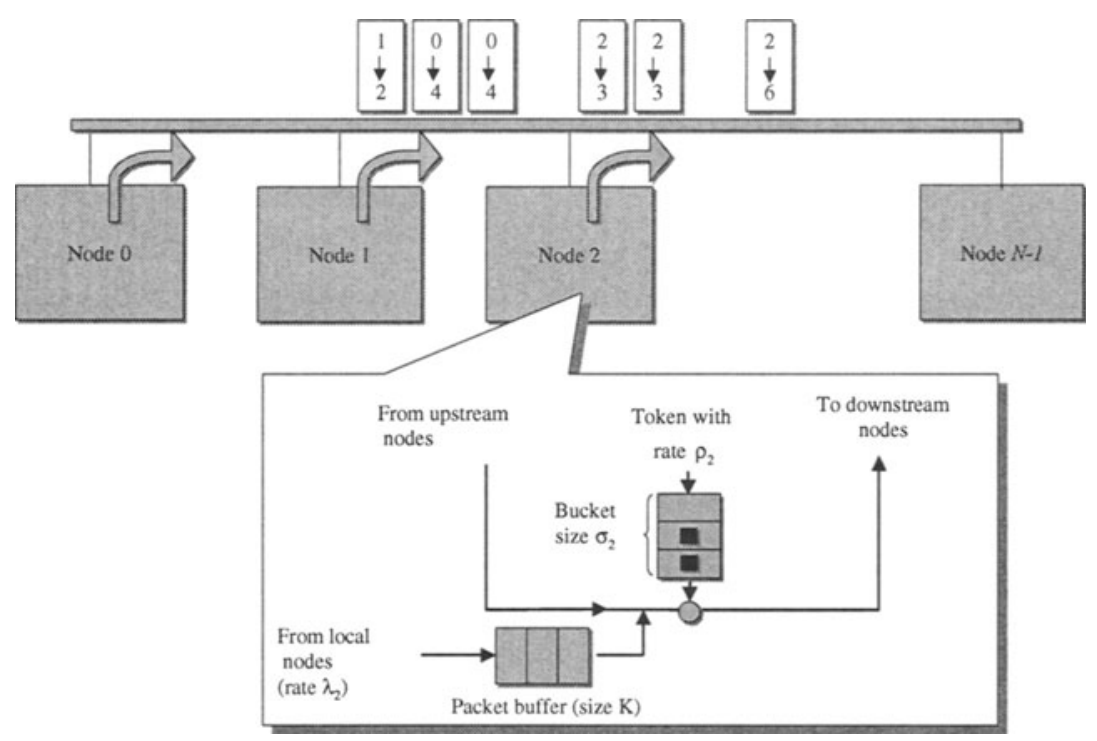

Fig.7 Flow enforcement by leaky-bucket

See Fig.7. Consider a slotted time axis where each slot is of length $1 / \rho$, and a new token is generated at each slot boundary. A newly arriving token joins the token pool if the pool contains fewer than $\sigma$ tokens; otherwise it is discarded. We will derive the steady-state joint probability distribution of the queue size and the token pool occupancy at slot boundaries, just prior to the generation of a new token. Note that we neglect an asynchronous operation of our system, but it is only for modelling purpose.

We introduce $P(m, i)$ the steady-state probability of having $m$ tokens in the token pool and $i$ packets in the packet buffer. The number of tokens cannot exceed $\sigma$, and the packet should wait in the buffer if and only if there exist no tokens in the token pool. Thus, we have

$$
P(m, i)=0, \quad m>\sigma ; 1 \leq m \leq \sigma \text { and } i \geq 1 .
$$

For convenience, we introduce $p_{i}$ as

$$
p_{i}=\left\{\begin{array}{c}
P(\sigma-i, 0), \quad 0 \leq i \leq \sigma \\
P(0, i-\sigma), \quad \sigma \leq i
\end{array}\right.
$$


As described in the above, packet arrivals at the local node are assumed to follow a Poisson process. We further assume that arrivals of transit packets from the upstream nodes destined for the downstream nodes also follow a Poisson process with rate $\lambda_{u}$, which is given by

$$
\lambda_{u}=\sum_{j=0}^{i-1} \sum_{k=i+1}^{N-1} \lambda_{j, k}
$$

for node $i$. We note here that in actual, we need to take account of the departure processes from the leaky-bucket at the upstream nodes, which is treated as an input process at the current node. However, since the variance of the departure process tends to become smaller than the input Poisson process by the leaky-bucket, we can estimate the worst case by the above assumption. We introduce $a_{i}$ and $b_{i}$ to represent the probabilities of $i$ arrivals during a slot from the local node and from the upstream nodes, respectively, i.e.,

$$
a_{i}=\frac{e^{-\lambda / \rho}(\lambda / \rho)^{i}}{i !} ; b_{i}=\frac{e^{-\lambda_{u} / \rho}\left(\lambda_{u} / \rho\right)^{i}}{i !}
$$

Further, we introduce $\bar{a}_{i}$ and $\bar{b}_{i}$ as

$$
\bar{a}_{i}=1-\sum_{j=0}^{i} a_{j} ; \bar{b}_{i}=1-\sum_{j=0}^{i} b_{j}
$$

Since we consider the finite capacity of the packet buffer (denoted by $K$ ), a packet that arrives and finds the buffer full is discarded. The transit packets are given higher priority since we assume to have no means to store the packets in an optical domain. Accordingly, we consider that the transit packet gets the token from the token pool at the local node and leaves the node. The steady-state equations are then given by

$$
\begin{gathered}
p_{i}=p_{0} \sum_{k=0}^{i} b_{k} a_{i-k}+\sum_{j=0}^{i} p_{j+1} \sum_{k=0}^{i-j} b_{k} a_{i-j-k,} \\
0 \leq i \leq \sigma+K-1 .
\end{gathered}
$$

The above equations can be solved by first assuming a value for $p_{0}$, and recursively computing $p_{\mathrm{i}}$ 's $(1<i<\sigma+K)$ using 


$$
p_{i}=\frac{1}{a_{0} b_{0}}\left(p_{i-1}-p_{0} \sum_{k=0}^{i-1} b_{k} a_{i-1-k}-\sum_{j=0}^{i-2} p_{j+1} \sum_{k=0}^{i-1-j} b_{k} a_{i-1-j-k}\right)
$$

All quantities are finally normalized with

$$
\sum_{i=0}^{\sigma+K} p_{i}=1
$$

The throughput at the designated node is then obtained as

$$
\begin{aligned}
& T=p_{0} \sum_{k=0}^{\sigma+K-1} b_{k}\left\{\sum_{i=1}^{\sigma+K-k} b_{k}+(\sigma+K-k) \bar{a}_{\sigma+K-k}\right\} \\
& +\sum_{j=1}^{\sigma+K} p_{j} \sum_{k=0}^{\sigma+K-1} b_{k}\left\{\sum_{i=1}^{\sigma+K-j-k+1} i a_{i}+(\sigma+K-j-k+1) \bar{a}_{\sigma+K-j-k+1}\right\}
\end{aligned}
$$

Finally, we obtain the packet loss probability at the node as

$$
P_{\text {loss }}=1-\frac{T}{\lambda / \rho}
$$

We can also derive waiting times for the packet stored at the buffer by applying the approach taken in [21], although we omit the result. In numerical examples, we will only show the maximum waiting time of the packets that are stored in the buffer without lost. It is simply given as

$$
W_{\max }=\sigma / \rho .
$$

We last note that we can also consider the bursty traffic such as an $\mathrm{ON}-$ OFF process, but for that purpose, we need a more extension. See, e.g., [22]. The numerical examples for the packet loss probability will be shown in Subsection 4.1.

\subsection{Optical implementation}

The protocol for accessing the line is shown in Fig.8. If the arriving packet at node $j$ has the destination label of node $j$, the packet is dropped from switch 1 . If the arriving packet has a different destination from node $j$, and if the token pool is nonempty, it is bypassed to downstream nodes. However, the arriving packet having a different destination from node $j$ is 
discarded when the token pool is empty. We will put a higher priority on the bypassing traffic than the traffic to be added from the node $j$, therefore, the packet transmission from node $j$ is awaited during the burst from node $i(<j)$ passes by node $j$.

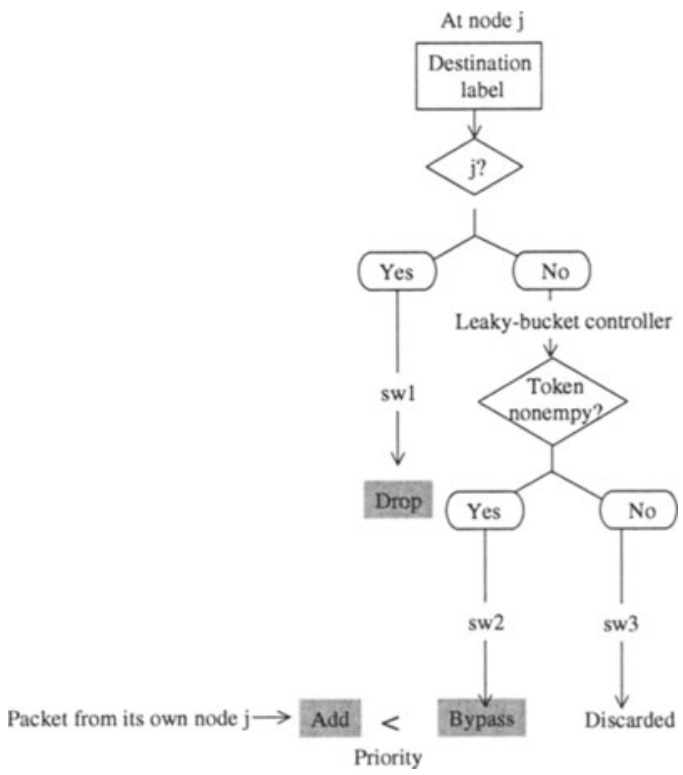

Fig.8 Access protocol of asynchronous rate-controlled packet transmission

The implementation of the P-ADM is shown in Fig.9. It consists of a photonic label selector, three $1 \times 2$ optical switches, two optical delay lines, and an electronic leaky-bucket controller. The photonic label selector directly controls optical switch 1 , and the control signal from it is also fed to the leaky-bucket controller. The leaky-bucket controller controls optical switches 2 and 3. The delay time $\tau$ of the optical delay line has to be set equal to the processing time required for photonic label selector, while, the delay time $\tau_{1}$ is set equal to the processing time required for the leaky-bucket controller. Optical switch 1 drops the arriving packet when it turns to the cross-state, otherwise it stays in the bar-state to bypass the packet. Optical switch 3 stays in the bar-state to bypass the arriving packet, and it turns to the cross-state to discard the arriving packet. Optical switch 2, controlled by the leaky-bucket controller, is allowed to turns to the cross-state to add the packet from the buffer only when there is no bypassing packet, otherwise it stays in the bar-state to bypass the packet. 
It is noted that the leaky-bucket control is very simple; only register fetch and add operations are necessary to implement the leaky-bucket mechanism. When we consider 100 Gbps transmission line and 1,000 bit-long packet as described in the numerical examples in 4.1 , a packet transmission time becomes $10 \mathrm{~ns}$. Thus, it is sufficient to execute several instructions for performing the leaky-bucket algorithm even by the current processor technology.

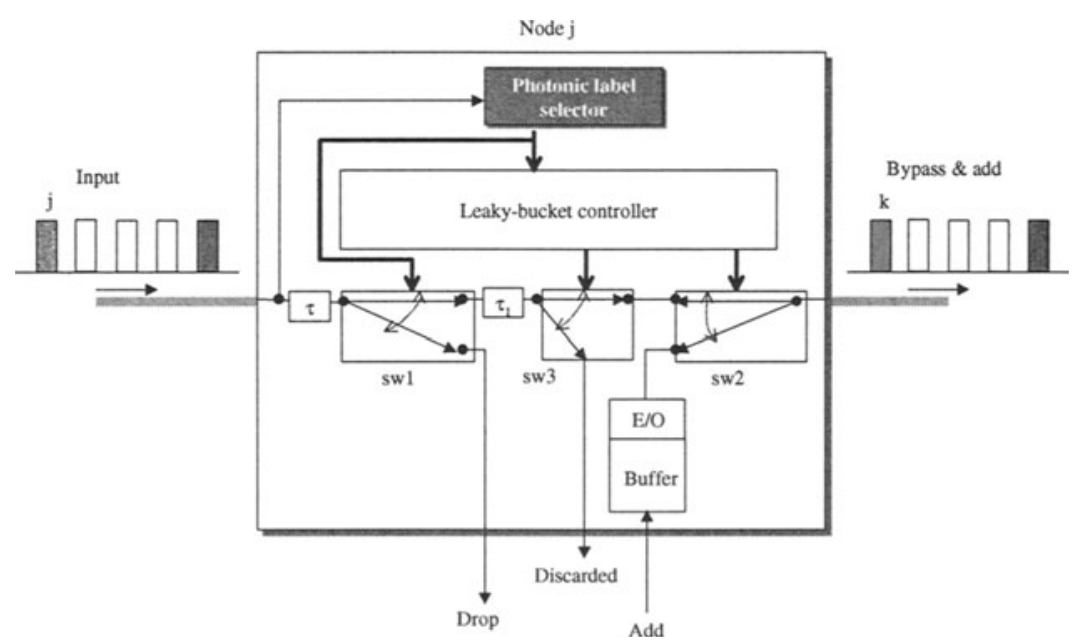

Fig.9 Optical implementation P-ADM for asynchronous rate-controlled packet transmission

\section{DISCUSSIONS}

\subsection{Numerical examples of rate-controlled asynchronous packet transmissions}

By using the analysis method in Subsection 3.1, we now provide several numerical examples. We consider the balanced traffic, i.e., $\lambda=\lambda_{i j}$ for all $(i, j)$ throughout this subsection. The line capacity $C$ and the packet length are fixed at $100 \mathrm{Gbps}$ and 1,000 bits, respectively. The token generation rate at node $i$ is determined by taking into account two kinds of traffic flows; ( $N-I$ 1) connections are originated at node $i$ and destined for the downstream 
nodes. Other $i(N-i-1)$ connections are transit flows which are originated at the upstream nodes and destined for the downstream nodes.

In Fig.10, we first show the packet loss probabilities dependent on the node position of the line for $N=12$. The target traffic load for each connection is set to be $C / 36$ where 36 is the total number of transit and originating connections at node 5. Three cases for the buffer size are shown; $K=10,15$ and 20. The token pool size (or bucket depth) $\sigma$ is set to be 10 . The traffic load is $2.08 \mathrm{Gbps}$ for each connection, which corresponds to $75 \%$ of the bandwidth allocated to each connection in the pseudo TDMA system. The packet loss probabilities are slightly increases at downstream nodes. It is because in the downstream node, the traffic from the upstream node becomes increased, and it suppresses the packet transmission from the local node. For example, in the case of the buffer size $K=20$, the maximum waiting time at the buffer ranges from $0.100 \mu$ s (at node 5) to $0.327 \mu$ s (at node 0 and 10). By considering the time duration of 1,000 bit-long packet is $0.01 \mu \mathrm{s}$ for the bit rate of $100 \mathrm{Gbit} / \mathrm{s}$, the waiting time would be reasonable.

We next show the packet loss probabilities dependent on the traffic load in Fig.11. The horizontal axis shows the traffic load at each node. We plot the worst case, i.e., the packet loss probabilities at $(N-2)$ nd node. The buffer size $K$ is changed as 10,15 and 20 in the figure. Last we show the packet loss probabilities dependent on the number of nodes on the line in Fig.12. For that purpose, we again fix the traffic load at $1.0 \mathrm{Gbps}$; identically set for all connections. Thus, as the number of total nodes is increased, the traffic load at each link gets large and the performance is degraded in both two cases. The packet buffer size is set to be 10,15 and 20 . The token pool size of all nodes is identically set to be 10 . All results are for $(N-2)$ nd node. 


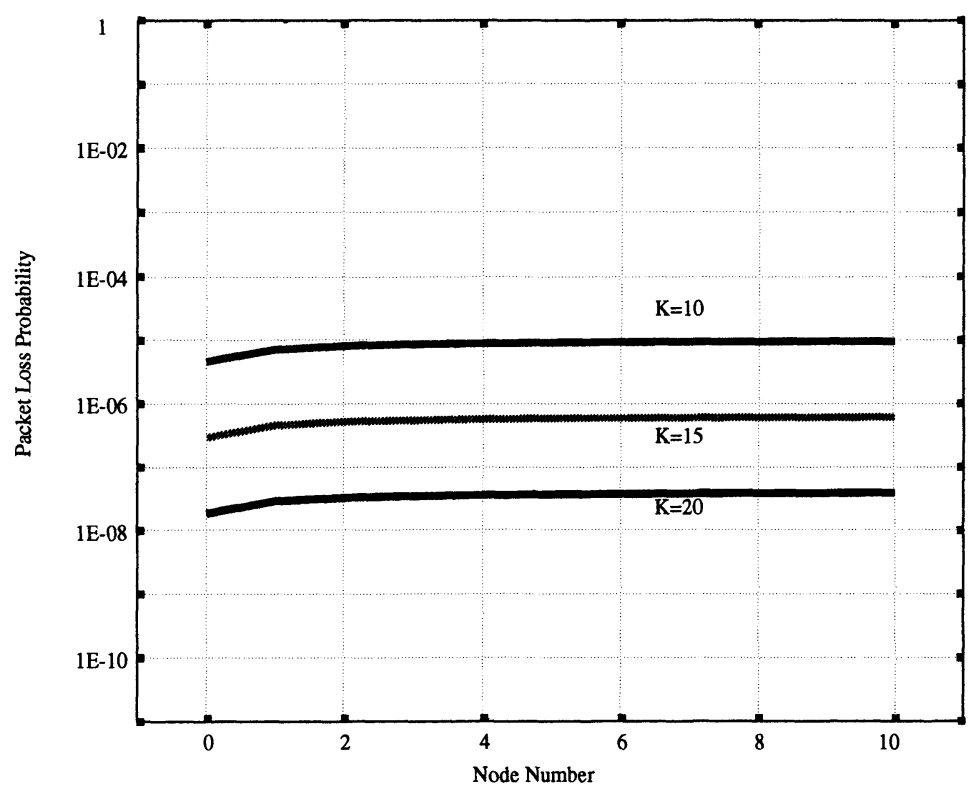

Fig.10 Packet loss probabilities at nodes

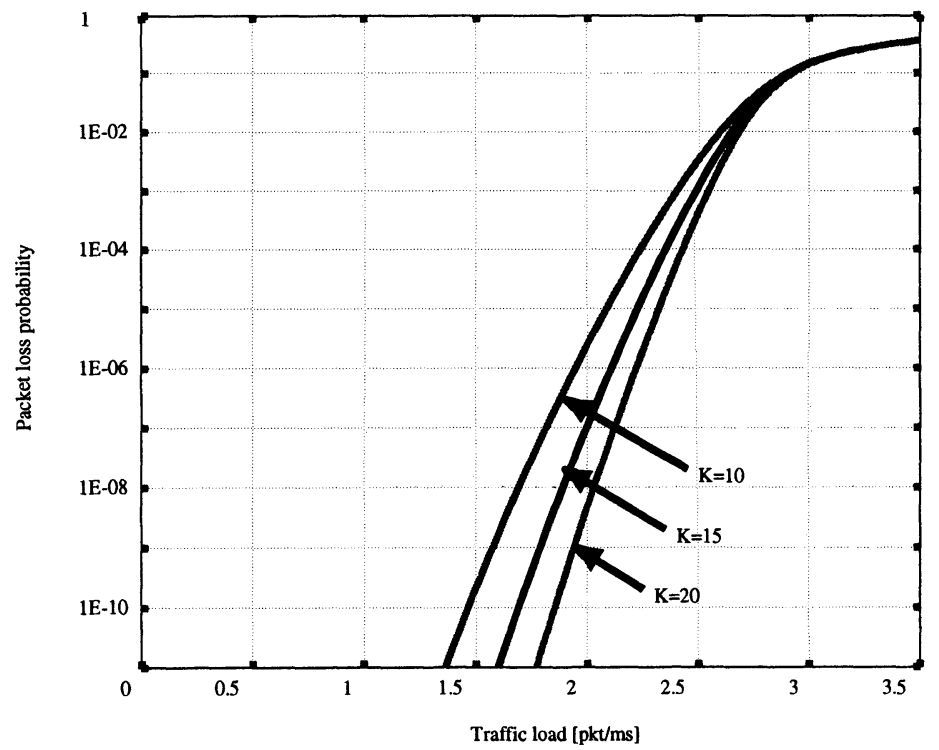

Fig.11 Packet loss probabilities vs. traffic load 


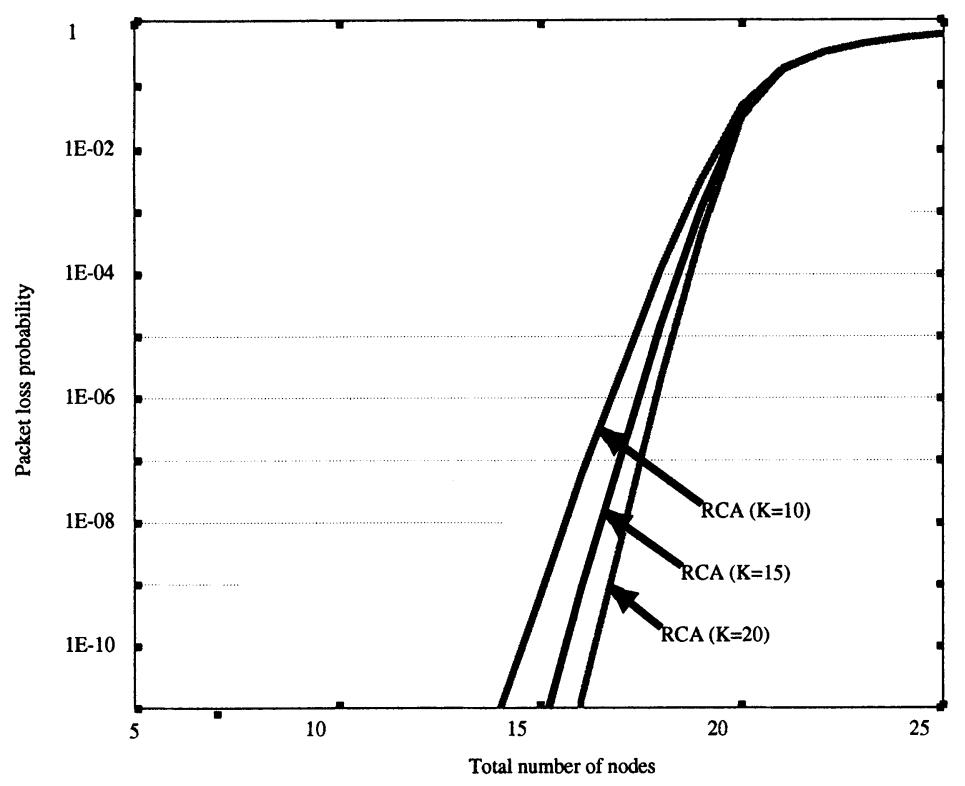

Fig.12 Packet loss probabilities vs. the number of nodes

\subsection{Perspective for ultrahigh-speed optical data net- working}

The rate-controlled access has a distinct advantage over conventional multiple access protocols because it adopts a sort of so-called open-loop control [23]. Another is a closed loop control where the control line (or control slot) is used to reserve the next transmission on the line. Such a control method can be found in, e.g., Distributed Queue Bus (DQDB). The minimum packet size in Ethernet has to be at least twice the propagation delay in bits so that the node will hear a collision before it stops sending and mistakenly assumes the transmission is successful. As increasing the bit rate, more and more bits can be packed into the fiber. For example, the minimum packet size for $100 \mathrm{Gbps}$ is $10^{5}$ bits for the propagation delay of only $100 \mathrm{~m}$-long optical fiber. Such a large packet size is impractical and therefore, the access techniques such as GbE (E: Ethernet), 10GbE, and DQDB will have a difficulty in achieving high performance for a long distance because their performance can be easily influenced by the transmission length. On the contrary, the performance of the rate-controlled access is by no means liable to the transmission length, and this characteristic can be exploited for ultra high-speed access. 
There is another an advantage in the rate-controlled access compared with optical TDMA (OTDMA), which is a multiple access protocol in optical layer [24]. An ultrahigh-speed OTDM has been realized up to a hundred Gbps [25]. All-optical regenerative OTDM add/drop multiplexing at 40Gbps has been materialized [26]. OTDMA is not as efficient for bursty data transmissions because it uses a time-slotted access protocol.

Ultimate performance of the rate-controlled asynchronous access will be predicted from a viewpoint of its optical implementation. The overall processing speed of P-ADM is limited by the capability of the photonic label selector, which is governed by the propagation delay required for the optical correlation. It has been experimentally demonstrated that optical packet consisting of photonic label using 8-chip-long optical code is processed, and optical packet of 64 bit-long payload data at $10 \mathrm{Gbit} / \mathrm{s}$ is routed via optical switch [14]. The processing capability reaches $29 \mathrm{Gpps}$ with the time duration of photonic label of 35ps. Assume that the packet length is 1,000 bit, PADM can be interfaced with the link of $29 \mathrm{Tbps}$. The accessible number of nodes depends upon the length of optical code. For the chip pulsewidth of $1 \mathrm{ps}$ the maximum code length will become $5 \mathrm{ps}$ for the bit rate of $100 \mathrm{Gbps}$. For 5-chip long optical code, the number of available optical codes are $2^{5-}$ $1=16$. Note that the factor of -1 is due to the elimination of symmetry code sequences.

\section{CONCLUSION}

Packet-selective photonic add/drop multiplexing (P-ADM) using photonic label selector based upon optical code correlation and its novel application to ultrahigh-speed optical data networking have been proposed. P-ADM provides a finer granularity of traffic than conventional $\lambda$-ADM, and it can handle the traffic on a packet-by-packet basis. The optical implementation PADM has been presented by using off-the-shelf optical devices such as FBG and optical gate switch, and the ultrahigh-speed processing capability as high as $29 \mathrm{Gpps}$ has been predicted. The application to optical data networking in LAN and MAN, the rate-controlled asynchronous access has been studied, and the numerical examples have confirmed performances of low packet loss probability.

Finally, another promising application of the packet-selective P-ADM is to the network protection and restoration. $\mathrm{P}-\mathrm{ADM}$ will be able to provide the protection to individual traffic on a wavelength path. Hence, this will provide with differentiated class of protections; each traffic has different priority of protection and restoration. 
References:

[1] C. A. Brackett, "Dense wavelength division multiplexing networks: Principles and applications," IEEEJ. Select. Areas in Commun., vol. 8, pp. 948-964, 1990.

[2] K. Sato, S. Okamoto, and H. Hamada, "Network performance and integrity enhancement with optical path layer technologies," IEEE J. Select. Areas in Commun., vol.12, pp.159170, 1994.

[3] P. Bonenfant and A. R.-Moral, "Optical data networking," IEEE Commun. Magazine, pp.63-70, March 2000.

[4] M. Murata, "Challenges for the next-generation Internet and the role of IP over photonic networks," to appear in IEICE Trans. Commun. vol.E83-B, No.8, 2000.

[5] E. Modiano and A. Narula-Tam, "Mechanism for providing optical bypass in WDM-based networks," Optical Networks, vol.1, pp.11-18, 2000.

[6] R. Ramaswami and K. N. Sivarajan, Optical Networks -A Practical Perspective-, S. F.: Morgan Kaufmann Publishers, Inc, 1998, ch.8.

[7] Y. Takushima and K. Kikuchi, "Photonic switching using spread spectrum technique," Electron. Lett., vol.30, pp.436-438, 1994.

[8] M.D. Vaughn and D.J. Blumenthal, "All-optical updating of subcarrier encoded packet headers with simultaneous wavelength conversion of baseband payload in semiconductor amplifiers," IEEE Photonic Technol. Lett., vol.9, pp.827-829, 1997.

[9] A. Carena, M.D. Vaughn, R. Gaudino, M. Shell, and D.J. Blumenthal, "OPERA: An optical packet experimental routing architecture with label switching capacity," $J$. Lightwave Technol., vol.16, pp.12135-2145, 1998.

[10] X. Jiang, X. P. Chen, and A.E. Willner, "All optical wavelength independent packet header replacement using a long $\mathrm{CW}$ region generated directly from the packet flag," IEEE Photonic Technol. Lett., vol.10, pp.1638-1640, 1999.

[11] M. C. Cardakli, D. Gurkan, S. A. Havstad, A. E. Willner, "Variable-bit-rate header recognition for reconfigurable networks using tunable fiber-Bragg-gratings as optical correlators," 2000 Optical Fiber Conference (OFC2000), TuN2 (Baltimore, 2000).

[12] W. I. Way, Y.-M. Lin, and G. -K. Chang, "A novel optical label swapping technique using erasable optical single-sideband subcarrier label," 2000 Optical Fiber Conference (OFC'99), WD6 (Baltimore, 2000).

[13] K. Kitayama and N. Wada, "Photonic IP routing," IEEE Photonic Technol. Lett., vol.11, pp.1689-1691, 1999.

[14] N. Wada and K. Kitayama, "Photonic IP routing using optical codes: 10Gbit/s optical packet transfer experiment," 2000 Optical Fiber Conference (OFC'99), WM51 (Baltimore, 2000).

[15] H. Sotobayashi and K. Kitayama, "Optical code based label swapping for photonic routing," to appear in IEICE Trans. Commun. vol.E83-B, No.8, 2000.

[16] J. Salehi, "Code division multiple access techniques in optical fiber networks-Part I: Fundamental principles," IEEE Trans. Commun., vol.37, pp.824-833, 1989.

[17] P. Prucnal, M. Santro, and T. Fan, "Spread spectrum fiber optic local area network using optical processing," IEEE/OSA J. Lightwave Technol., vol.4, pp.307-314, 1986.

[18] K. Kitayama, "Code division multiplexing lightwave networks based upon optical code conversion," IEEE Selected Areas in Commun., vol.16, pp.1309-1319, 1998.

[19] K. Kitayama, H. Sotobayashi, and N. Wada, "Optical code division multiplexing (OCDM) and its applications to photonic networks (INVITED)," IEICE A., vol.E82-A, pp.2616-2626, 1999.

[20] P.C. The, P. Petropoulos, M. Ibsen, and D. J. Richardson, "A 10Gb/s, 160Gchips/s OCDMA coding: decoding system based on superstructured fiber gratings," 2000 Optical Fiber Communication Conference (OFC2000), PD9 (Baltimore, March 2000). 
[21] M. Sidi, W.-Z. Liu, I. Cidon, and I. Gopal, "Congestion control through input rate regulation," IEEE Transactions on Communications, vol. 41, March 1993.

[22] M. Murata, Y. Ohba, and H. Miyahara, "Analysis of flow enforcement algorithm for bursty traffic in ATM networks," Proceedings of IEEE INFOCOM '92 (Florence, Italy), pp. 2453-2462, May 1992.

[23] H. Ohsaki, M. Murata, H. Suzuki, C. Ikeda, and H. Miyahara, "Rate-based congestion control for ATM networks," ACM SIGCOMM Computer Communication Review, vol. 25, pp. 60-72, April 1995.

[24] S-. W. Seo, K. Bergman, and P. R. Prucnal, "Transparent optical networks with timedivision multiplexing," IEEE J. Selected Areas in Commun./J. Lightwave Technol., vol.14, pp.1039-1051, 1996.

[25] S. Kawanishi, "120Gbit/s OTDM system prototype," 24th European Conference on Optical Communications (ECOC'98)), PD, p.41 (Madrid 1998)

[26] A. Buxens, A. T. Clausen, H. N. Poulsen, P. Jeppesen, St. Fischer, M. Dulk, E. Gamper, W. Vogt, W. Hunziker, E. Gini, and H. Melchior, "All-optical regenerative OTDM add/drop multiplexing at 40Gbit/s using monolithic InP Mach-Zehnder interferometer," CLEO2000, CWD3, 2000. 